\title{
The picture-word interference effect is not a Stroop effect
}

\author{
R. DeLl'ACQua \\ Università di Padova, Padova, Italy \\ R. JoB \\ Università di Trento, Rovereto, Italy \\ F. Peressotti \\ Università di Padova, Padova, Italy \\ AND \\ A. Pascali \\ Università di Ferrara, Ferrara, Italy
}

\begin{abstract}
A psychological refractory period (PRP) paradigm was used to isolate the locus of the picture-word interference effect along the chain of processes subtended in name production. Two stimuli were presented sequentially on each trial, separated by a varying stimulus onset asynchrony (SOA). The first stimulus, $\mathrm{S} 1$, was a tone that required a manual response. The second stimulus, S2, was a picture-word stimulus associated with picture naming. The distractor word was conceptually related to the picture on half of the trials, and unrelated in the other trials. A picture-word interference effect was found at long SOA, but not at short SOA. Such underadditive interaction between SOA and semantic relatedness suggests strongly that the locus of the picture-word interference effect is functionally earlier than the PRP effect locus. The results are discussed in relation to models of word production suggesting the involvement of central mechanisms in the selection of lexical output.
\end{abstract}

It is widely agreed that the locus of the Stroop effect along the chain of mental processes subtended in the color name production is a "late," postperceptual locus (see MacLeod, 1991, for a comprehensive review of Stroop models). Extant explanations of the Stroop effect revolve around the idea that a response code based on the word color and a response code based on the word meaning are generated in parallel, and that such codes interfere mutually during response selection. Support for this view has been provided by Fagot and Pashler (1992; Experiment 7) using a variant of the psychological refractory period (PRP) paradigm. These authors had subjects perform a speeded manual response (R1) to an auditory stimulus (S1) followed, at a varying stimulus onset asynchrony (SOA), by a Stroop word (S2) associated with a speeded color naming response (R2). Two distinct RTs were recorded on each trial, one to the S1 (RT1), and one to S2 (RT2). As is pervasively observed in similar PRP circumstances (see Pashler, 1994a, for a review), RT1 was minimally affected by SOA, whereas RT2 increased substantially as SOA was decreased. The color-word congruency manipulation produced a predictable Stroop effect on color naming responses, with longer RT2 to incongruent stimuli relative to RT2 to congruent stimuli. Interestingly, color-word con- gruency and SOA produced effects on RT2 that were additive, that is, the Stroop effect was constantly observed at all tested S1-S2 SOAs (i.e., -50, 50, 150, and $450 \mathrm{msec}$ ).

The conclusion based on these findings is reproduced graphically in Figure 1, where the processing required for the first task (i.e., the speeded manual response to S1) and for the second task (i.e., the speeded naming response to S2) of the PRP paradigm has been broken down into the stages of perceptual encoding (PE), response selection (RS), and response execution (RE).

The model relies on the assumption that response selection is a mental operation that suffers significant capacity limitations when two tasks must be performed in rapid succession. Response selection in this view has been conceived of as a "bottleneck" in the processing required by overlapping tasks (Pashler \& Johnston, 1989; see also Pashler 1994b).

Some researchers (e.g., Tombu \& Jolicœur, 2003, 2005) have proposed that, at least on particular occasions, central processing capacity may be allocated in a graded fashion between tasks, namely, without invoking an immutable bottleneck in multitasking processing. Others researchers have proposed that the PRP effect arises as a consequence of the subjects' strategy to defer portions of the processing 


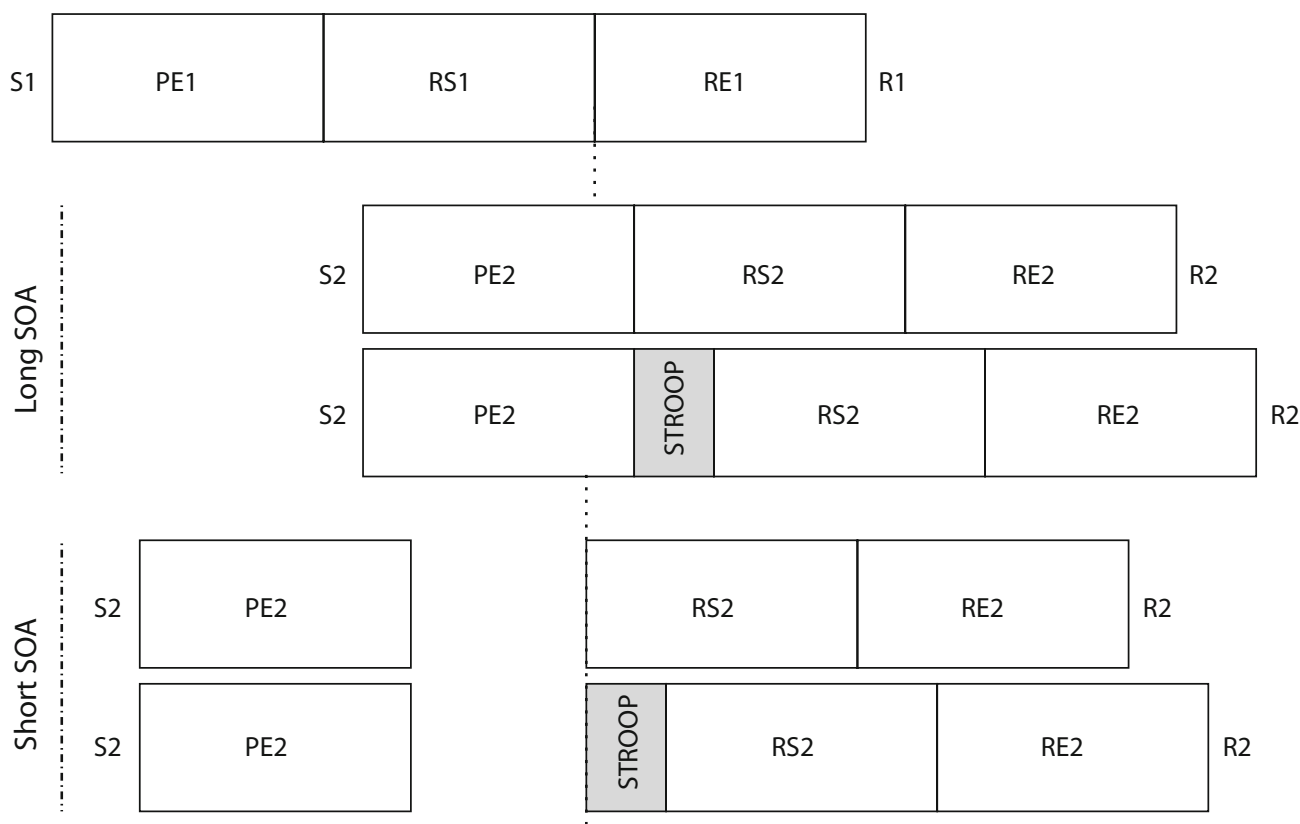

time

Figure 1. Gant diagrams illustrating an account of the additivity between SOA effect and Stroop effect in the context of a psychological refractory period (PRP) paradigm. In the boxes, $\mathrm{PE}=$ perceptual encoding, $\mathrm{RS}=$ response selection, $\mathrm{RE}=$ response execution. The shaded box marked with the label "STROOP" indicates a locus of the Stroop effect compatible with the conclusions of Fagot and Pashler (1992; Experiment 7; see text for details).

required for either task in a multitasking condition in order to limit the possibility to incur in an error (e.g., Meyer \& Kieras, 1997). In the General Discussion, we will examine the general implications of such views in the context of the results produced by the present investigation.

At short SOAs, the limitation in performing response selection for more than one incoming stimulus at a given time results in the postponement of the response selection in the second task (RS2) until after the response selection in the first task (RS1) is completed. The notion incorporated in the model of Figure 1 is that the locus of the Stroop effect (indicated by the shaded box labeled "STROOP") is at response selection, causing a general prolongation of RT2 when the word color and the word meaning are incongruent. PRP effect apart, the model accounts successfully for the additivity of SOA and the Stroop manipulation, given that prolonging any processing stage at or after response selection in the second task is hypothesized to simply add a temporal quantity to RT2 that remains constant across SOAs. Incidentally, the model also fits nicely with the positive correlation between RT1 and RT2 that is generally found in PRP results. As the model in Figure 1 makes clear graphically, this is hypothesized to occur based on the observation that the sooner response selection in the first task finishes, the sooner response selection in the second task can commence.

The picture-word interference (PWI) effect is the slowing of picture naming time usually observed when a picture (e.g., bed) is displayed concurrently with a conceptually related word (couch) relative to when the picture is displayed with a conceptually unrelated word (tomato). The current debate about the locus of the PWI effect along the chain of mental processes leading to word production is dominated by positions that diverge radically. Some authors (e.g., Levelt et al., 1999) have proposed that the PWI effect arises at the level of lexical selection. A relative activation account is invoked in this framework to explain PWI effect. Succinctly, the higher the cumulative activation of lexical nodes competing with the lexical node corresponding to the picture name, the longer the time taken to produce the picture name. When the distractor word is conceptually related to the picture, the distractor lexical node receives activation from both the word itself and the picture, increasing the word level of activation, and thus the competition for selection of the target lexical node. Other authors (e.g., Costa, Alario, \& Caramazza, 2005; Miozzo \& Caramazza, 2003) have instead proposed that PWI effect depends critically on the speed with which a postlexical phonological buffer is freed from distractor information. This position is supported principally by the counterintuitive negative correlation between the lexical frequency of distractor words and PWI effect magnitude.

These models will be discussed in more detail in the final Discussion section. For the current purposes, it suffices to note that it is not infrequent in articles focused on the PWI effect to come across definitions of the pictureword interference task in terms of a "Stroop-like task." (e.g., Finkbeiner \& Caramazza, 2006). For some researchers at least, this probably engenders from no more than a habit. Other researchers invoke noncoincidental similari- 
ties between the two effects that make them functionally comparable at a number of levels. For instance, it is well know that PWI effect vanishes if subjects are instructed to name the word surrounded by the picture. This is held to bear a close analogy with the absence of the Stroop effect when subjects have to read the word instead of naming the color. Furthermore, the manipulation of SOA (i.e., the temporal lag between the color and word dimensions in the Stroop task, and between the picture and the embedded word in the picture word interference task) produces virtually identical results in the two tasks. Whereas SOA does not play any evident modulatory role when subjects read the word, SOA generates a highly peculiar variation of Stroop and PWI effects as the SOA is varied in a range of $-400 \mathrm{msec}$ (the word leads the color/picture) to $400 \mathrm{msec}$ (the word trails the color/picture). When the word onset falls within $100 \mathrm{msec}$ of the color/picture, both the Stroop effect and the PWI effect are usually manifest. However, when the word onset occurs $400 \mathrm{msec}$ before the color/picture, both effects turn to facilitation (e.g., Bloem, van den Boogaard, \& La Heij, 2004; Glaser \& Düngelhoff, 1984).

The aim of the present work was to use a variant of the PRP task to test whether the similarity between the Stroop effect and the PWI effect generalizes to the PRP conditions illustrated above. In the present empirical context, subjects were instructed to make a speeded manual response (R1) to an auditory stimulus (S1), followed at a varying SOA, by a picture-word compound stimulus (S2) associated with a speeded picture naming response (R2). If the two effects originated for causes that are functionally analogous (i.e., both effects originating at or after the response selection stage), we reasoned that we would find a PRP effect affecting RT2, a positive correlation between the RT1 and RT2, and, critically, the additivity of SOA and PWI effects on RT2, as found by Fagot and Pashler (1992) for the Stroop effect.

\section{METHOD}

\section{Participants}

Fourteen students of the University of Padova, nine males, mean age 24 years, volunteered to participate in the experiment. All had normal or corrected-to-normal vision, and normal hearing.

\section{Apparatus and Stimuli}

Forty-eight line drawings of real-world concepts with a high name agreement $(\mathrm{H}=.01$; Snodgrass \& Yuditsky, 1996) were selected from Dell'Acqua, Lotto, and Job's (2000) database. Each picture was paired with two distractor words, one within-category conceptually related word and one conceptually unrelated word. The distractor words were not part of the response (picture names) set. The two word sets were matched for number of letters, number of syllables, lexical frequency, concept familiarity, concept typicality, and age of acquisition (all $t \mathrm{~s}<1$ ). Equiluminant pictures and words were displayed in white $\left(45 \mathrm{~cd} / \mathrm{m}^{2}\right)$ against the black background $\left(8 \mathrm{~cd} / \mathrm{m}^{2}\right)$ of a cathode-ray tube monitor, controlled by an IBM-clone and MEL software. The words were displayed at the center of the monitor in Romantri 32 font, surrounded by the pictures that could all be inscribed in a square portion of the monitor measuring $6^{\circ} \times 6^{\circ}$. The same apparatus was used to control vocal/manual response recordings, and the generation of the acoustic stimuli, pure tones at frequencies of $300,600,1200 \mathrm{~Hz}$, presented through the built-in speaker of the computer with the volume set to be always clearly audible.

\section{Design and Procedure}

Each trial began with the presentation of a fixation point at the center of the monitor that lasted $1,000 \mathrm{msec}$. The offset of the fixation point was followed by a blank interval of $800 \mathrm{msec}$, and by the presentation of a randomly selected tone (S1) for $50 \mathrm{msec}$. The participants were instructed to rest the index, medium, and annular fingers of the right hand on three horizontally arrayed keys of a response box, and to press one of the keys according to the tone pitch. The instructions stressed the importance to be always as fast and accurate as possible in making the response to the tone. At an SOA of 100,350 , or 1,000 msec, a picture-word compound stimulus (S2) was displayed. The participants were instructed to name the picture as fast and accurately as possible while ignoring the word, using a microphone placed before their mouth. The experimental list of 288 stimuli (i.e., 48 pictures $\times 2$ words $\times 3$ SOAs) was randomized for each participant, and organized at runtime in 12 blocks of 24 trials each, that were preceded by a block of 24 stimuli that were not included in the experimental list. In each block, the levels of SOA and picture-word conceptual relatedness were equiprobable.

\section{RESULTS}

Two reaction time responses were recorded on each trial, one to S1 (RT1) and one to S2 (RT2). Correct RT1 and RT2 were screened for outliers using the procedure described by Van Selst and Jolicœur (1994b), that led to the rejection of $1.2 \%$ of RT1s and $2 \%$ of RT2s. Mean RT1 and RT2, and the proportion of correct responses in Task1 and in Task2, were submitted to analysis of variance (ANOVA) in which SOA and conceptual relatedness were treated as within-subjects factors. A summary of the results is reported in Figure 2.

The left panel shows that RT1 was not affected by SOA, nor by semantic relatedness $\left(F_{\mathrm{S}}<1\right)$. In contrast, RT2 increased substantially as SOA was decreased $[F(2,26)=$ $85.7, p<.001]$; RT2 was generally longer with a conceptually related word than with an unrelated word $[F(1,13=$ $7.8, p<.03]$. SOA and relatedness however interacted significantly in the analysis of RT2 $[F(2,26)=10.5, p<$ $.001]$, the PWI effect that was observed at the longest SOA decreased progressively - from $68 \mathrm{msec}$ to $-7 \mathrm{msec}$ - as SOA was reduced. The analyses on the proportion of errors (.03 in Task1, .05 in Task2) did not reveal any significant effect $(F \mathrm{~s}<1)$. The right panel shows the expected dependency $[F(6,78)=16.3, p<.001]$ between RT1 and the PRP effect. For each participant, in each cell of the design, mean RT2s were computed based on RT1s divided into quartile bins (Q1 = shortest RT1; Q4 = longest RT1). As RT1 lengthened, the PRP effect increased.

\section{DISCUSSION}

The present results can be summarized as follows. A PWI effect was found that was underadditive with SOA in a classical PRP design. Whereas the PWI effect was fully manifest in the canonical form (i.e., longer RT2 to pictures coupled with conceptually related words than to pictures coupled with conceptually unrelated words) at the longest SOA in 
the present PRP design, the effect magnitude decreased progressively as SOA was decreased, up to a virtual absence of the PWI effect under conditions of maximal temporal overlap between the tasks - that is, at the shortest SOA.

The implications of the present findings involve different aspects of the models mentioned in the Introduction. The present findings are obviously incompatible with the often reiterated principle that the PWI effect comes about for limitations of the cognitive system that are analogous to those causing the Stroop effect (e.g., Roelofs, 2003). Whereas the Stroop effect has been shown to be additive with SOA effects in a PRP design (Fagot \& Pashler, 1992), the PWI effect was underadditive with SOA effects in the present PRP context.

The present results suggest strongly that the cause of the PWI effect must be assumed to be prior to the PRP effect locus. Given the large consensus on the notion that the main limiting factor in performing simultaneous tasks is response selection, a further conclusion is that the PWI effect originates prior to response selection. A potential account of the present findings that is in line with the foregoing considerations is graphically reproduced in Figure 3.

At long SOA, that is, under conditions of minimal (or nil) task overlap, the PWI effect results in the prolongation of RT2 when picture and word are conceptually related. At short SOA, the Gant diagrams makes clear that the conflict reflected in the PWI effect is resolved during the period in which response selection in the second task (RS2) waits for response selection in the first task (RS1) to be finished.

The present results have also implications for models of language production and for the general understand- ing of the causes of the PWI effect. Using a PRP paradigm, Ferreira and Pashler (2002) have provided evidence for the involvement of central processing mechanisms in lexical selection. Central processing mechanisms are the mental mechanisms hypothesized to be engaged by serial operations that include, besides response selection, mental rotation (Van Selst \& Jolicœur, 1994a), the look-up from long-term memory (Carrier \& Pashler, 1995), and the short-term consolidation of visual information (Jolicœur \& Dell' Acqua, 1998). Participants in Ferreira and Pashler's (2002) study were exposed to a first stimulus that was a picture-word compound associated with a speeded naming time response (RT1), followed, at a varying SOA, by a second stimulus composed of a pure tone associated with a three-alternative forced-choice reaction time response (RT2). As expected, RT2 increased as SOA was decreased, the ubiquitous PRP effect. Importantly, however, at short SOAs, the PWI effect observed in RT1 propagated millisecond by millisecond onto RT2, suggesting that a central stage involved in selecting the proper picture name for $\mathrm{S} 1$ interfered with the selection of the response for S2.

In light of the findings reported by Ferreira and Pashler (2002) suggesting that central mechanisms are involved both in response selection and in lexical selection, a further conclusion inspired by the present findings is that lexical selection accounts of PWI effect (e.g., Levelt et al., 1999; see also Roelofs, 1992) can be hardly reconciled with the present empirical scenario. A central locus of the PWI effect would indeed be incompatible with the underadditivity of PWI effects and SOA effects observed in the present context. On the other hand, however, PWI
Semantic Interference Across SOAs

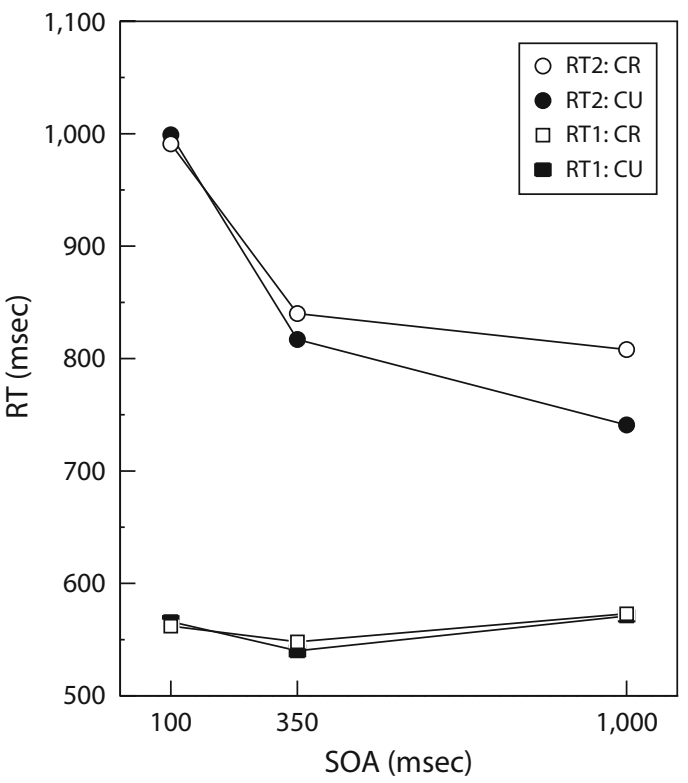

Dependency of RT2 on RT1

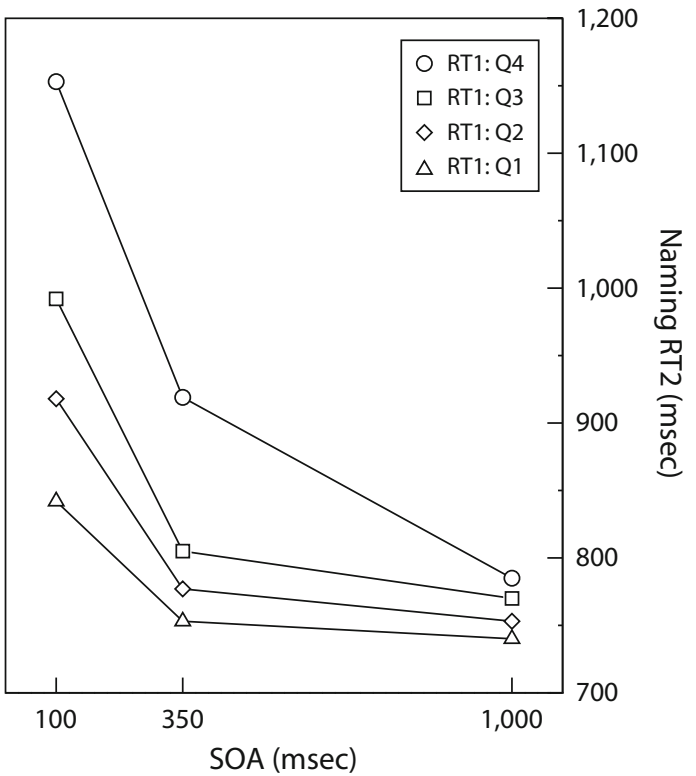

Figure 2. Left panel: Mean reaction times, plotted separately for Task1 $(\mathrm{RT} 1 ; \mathrm{S} 1=$ tone, $\mathrm{R} 1=$ manual) and Task2 (RT2; S2 = picture and word superimposed, $\mathrm{R2}=$ vocal response), as a function of semantic relatedness, and as a function of S1-S2 SOA. CR = picture and word conceptually related; $C U$ = picture and word conceptually unrelated. Right panel: Mean picture naming times (RT2) conditionalized on the speed of carrying out Task1 (RT1). In the graph, Q1 to Q4 indicate RT1 quartile, from the first (Q1) to the fourth (Q4) RT1 quartile. 


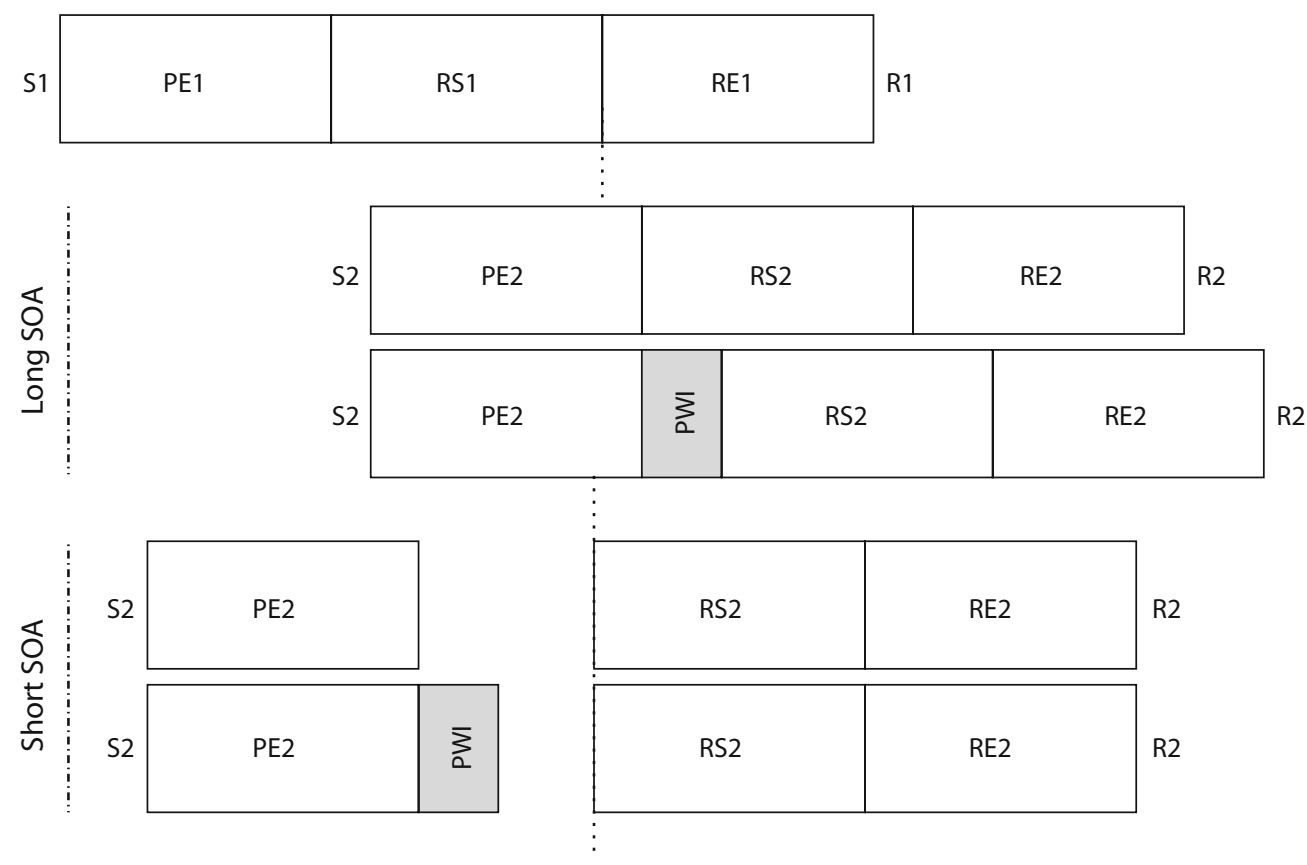

time

Figure 3. Gant diagrams illustrating an account of the underadditivity between SOA effect and PWI effect observed in the present empirical context. In the boxes, $\mathrm{PE}=$ perceptual encoding, $\mathrm{RS}=$ response selection, $\mathbf{R E}=$ response execution. The shaded box marked with the label 'PWI' indicates the supposed locus of the PWI effect.

models that do not rely on the notion of competition at the level of lexical selection do not appear to be more feasible in this respect either. A model like that proposed by Miozzo and Caramazza (2003), for instance, attributes bandwidth-limited properties in language production to a pre-articulatory buffer where the phonological codes generated on the basis of information conveyed by the word and the picture converge prior to the emission of a vocal response. The PWI effect in this model is predicated on the basis of the speed with which this buffer is freed by the interfering word code before the production of the picture name. More specifically, the picture name is hypothesized to be actively blocked as long as the distractor code lingers in the output buffer. Two are the options that must be considered in relation to this model. One option is that the picture name blocking engages central mechanisms. The other option, which seems to be the one favored by the authors (Miozzo \& Caramazza, 2003, p. 246) is to concede that competition at the level of lexical selection may sometimes occur under PWI conditions. Both options, to note, are however incompatible with a pre-central locus of the PWI effect. A suggestion based on the present findings is that competing semantic codes, though generated on the basis of information that differs in visual format (e.g., orthographic vs. pictorial), may start to interact very early in processing, prior to more central stages of processing, as we have recently put forth in the attentional blink field (Dell' Acqua, Jolicœur, Pascali, \& Pluchino, 2007; Potter et al., 2005). Positing a pre-central locus of semantic interplay between pictures and words in a PWI task (see
Figure 3) seems at present the only avenue suited to provide a consistent account of the present findings.

A comment is in order concerning possible alternative models of the PRP effect, and the implications of a different theoretical stance at this level on the present conclusions. It must be noted that the claim that Stroop effects and PWI effects originate from functionally different sources is largely independent from the particular model chosen to interpret the PRP effect. Tombu and Jolicœur (2003, 2005) have recently proposed that central capacity sharing may constitute a potential alternative to more traditional bottleneck models. Models like that of Tombu and Jolicœur have been advanced primarily with the scope to explain the contamination of RT1 by manipulations implemented in Task2, which is sometimes observed in PRP results. In the present case, hints of such contamination - and therefore of resource sharing - were absent in the RT1 results (i.e., no SOA effects on RT1; no effects of semantic relation on RT1). Obviously, this by no mean implies that the class of central capacity sharing models cannot account for the present findings, especially because bottleneck models of the PRP are held to be particular instances of central capacity sharing (i.e., when no sharing between overlapping tasks occurs, as was likely in the present case). Meyer and Kieras's (1997) model makes a set of articulate predictions on how subportions of the processing required for Task2 in a PRP design may be "locked out" depending on the type of strategy (more or less daring) that subjects adopt when performing in multitasking conditions. Although this model has been severely 
criticized, and some crucial predictions derived from it disconfirmed (Ruthruff, Johnston, \& Van Selst, 2001; Tombu \& Jolicœur, 2004), we cannot exclude that some ad hoc combination of lock-out points and resuming flags in the processing required to perform the present two tasks may be generated that would eventually fit the present results. The point we are trying to make here is, however, very specific. To reiterate, independently on the particular assumptions concerning the cause of the PRP effect, the conclusion that Stroop effects and PWI effects originate from distinct functional source seems inescapable.

An interesting aspect of the difference between PWI and Stroop causes relates to the timecourse of semantic activation elicited by distracting words in the two paradigms. One may indeed propose that PWI effects dissipate at short SOA because the word semantic code activation, and its consequent impact on picture naming speed, tends to decay over time, while the processing in Task 2 is waiting for response selection mechanisms to be freed by Task1. It is obvious that this hypothesis, without further assumptions, would nonetheless leave unexplained why decay of the distracting word meaning occurred in the present PRP design using a PWI Task2, and not in Fagot and Pashler's (1992) PRP design using a Stroop Task2. One way to salvage this view would be to resort to the idea that semantic activation mediated by color words and semantic activation mediated by real-world concepts differ in terms of temporal persistence. Until evidence in this direction is lacking, we favor an interpretation of the present findings that points to the functional dissociation of the sources of Stroop and PWI effects.

\section{AUTHOR NOTE}

Correspondence concerning this article should be addressed to R. Dell'Acqua, Dipartimento di Psicologia dello Sviluppo e della Socializzazione, Via Venezia, 8, 35131 Padova, Italy (e-mail: dar@unipd.it).

\section{REFERENCES}

Bloem, I., van den Boogaard, S., \& La HeiJ, W. (2004). Semantic facilitation and semantic interference in language production: Further evidence for the conceptual selection model of lexical access. Journal of Memory \& Language, 51, 307-323.

Carrier, L. M., \& PAshler, H. (1995). Attentional limits in memory retrieval. Journal of Experimental Psychology: Learning, Memory, \& Cognition, 21, 1339-1348.

Costa, A., Alario, F.-X., \& Caramazza, A. (2005). On the categorical nature of the semantic interference effect in the picture-word interference paradigm. Psychonomic Bulletin \& Review, 12, 125-131.

Dell'Acqua, R., Joliceur, P., Pascali, A., \& Pluchino, P. (2007). Short-term consolidation of individual identities leads to lag-1 sparing. Journal of Experimental Psychology: Human Perception \& Performance, 33, 593-609.

Dell'Acqua, R., LotTo, L., \& Job, R. (2000). Naming times and standardized norms for the Italian PD/DPSS set of pictures: Direct comparisons with American, English, French, and Spanish published databases. Behavior Research Methods, Instruments, \& Computers, 32, 588-615.

FAGOT, C., \& Pashler, H. (1992). Making two responses to a single object: Implications for the central attentional bottleneck. Journal of
Experimental Psychology: Human Perception \& Performance, 18, 1058-1059.

FerReIRA, V. S., \& PAShler, H. (2002). Central bottleneck influences on the processing stages of word production. Journal of Experimental Psychology: Learning, Memory, \& Cognition, 28, 1187-1199.

FinkBEINER, M., \& CARAMAZZA, A. (2006). Now you see it, now you don't: On turning semantic interference into facilitation in a Strooplike task. Cortex, 42, 790-796.

Glaser, W. R., \& DüNGelHoFf, F.-J. (1984). The time course of picture-word interference. Journal of Experimental Psychology: Human Perception \& Performance, 10, 640-654.

Jolicceur, P., \& DEll'ACQUA, R. (1998). The demonstration of shortterm consolidation. Cognitive Psychology, 36, 138-202.

LeVelt, W. J. M., Roelofs, A., \& MeYER, A. S. (1999). A theory of lexical access in speech production. Behavioral \& Brain Sciences, 22, 1-75.

MACLEOD, C. M. (1991). Half a century of research on the Stroop effect: An integrative review. Psychological Bulletin, 109, 163-203.

Meyer, D. E., \& Kieras, D. E. (1997). A computational theory of executive cognitive processes and human multiple-task performance: Part II. Accounts of psychological refractory-period phenomena. Psychological Review, 104, 749-791.

Miozzo, M., \& Caramazza, A. (2003). When more is less: A counterintuitive effect of distractor frequency in the picture-word interference paradigm. Journal of Experimental Psychology: General, 132, 228-252.

PASHLER, H. (1994a). Dual-task interference in simple tasks: Data and theory. Psychological Bulletin, 116, 220-244.

PASHLER, H. (1994b). Graded capacity-sharing in dual-task interference? Journal of Experimental Psychology: Human Perception \& Performance, 20, 330-342.

PASHLER, H., \& JOHNSTON, J. C. (1989). Chronometric evidence for central postponement in temporally overlapping tasks. Quarterly Journal of Experimental Psychology, 41A, 19-45.

Potter, M. C., Dell'Acqua, R., Pesciarelli, F., Job, R., Peressotti, F., \& O'Connor, D. (2005). Bidirectional semantic priming in the attentional blink. Psychonomic Bulletin \& Review, 12, 460-465.

Roelofs, A. (1992). A spreading-activation theory of lemma retrieval in speaking. Cognition, 42, 107-142.

RoELOFS, A. (2003). Goal-referenced selection of verbal action: Modeling attentional control in the Stroop task. Psychological Review, 110, 88-125.

RUThrufF, E., Johnston, J. C., \& VAn Selst, M. (2001). Why practice reduces dual-task interference. Journal of Experimental Psychology: Human Perception \& Performance, 27, 3-21.

SNODGRASS, J. G., \& YUDITSKY, T. (1996). Naming times for the Snodgrass and Vanderwart pictures. Behavior Research Methods, Instruments, \& Computers, 28, 516-536.

ToMBU, M., \& JoLICEUR, P. (2003). A central capacity sharing model of dual task performance. Journal of Experimental Psychology: Human Perception \& Performance, 29, 3-18.

ToMBU, M., \& JoLICEUR, P. (2004). Virtually no evidence for virtually perfect time-sharing. Journal of Experimental Psychology: Human Perception \& Performance, 30, 795-810.

ToMBU, M., \& JolicEUR, P. (2005). Testing the predictions of the central capacity sharing model. Journal of Experimental Psychology: Human Perception \& Performance, 31, 790-802.

VAN SELST, M., \& JOLICEUR, P. (1994a). Can mental rotation occur before the dual-task bottleneck? Journal of Experimental Psychology: Human Perception \& Performance, 20, 905-921.

VAN SELST, M., \& JOLICCEUR, P. (1994b). A solution to the effect of sample size on outlier elimination. Quarterly Journal of Experimental Psychology, 47A, 631-650.

(Manuscript received July 27, 2006; revision accepted for publication October $3,2006$. 